\title{
RANDOMISED CONTROLLED TRIALS
}

\author{
JENNIFER EVANS \\ London
}

From the epidemiologist's armchair, randomised controlled trials (RCTs) are the most straightforward of studies to design and interpret because of their unique ability (if properly conducted) to eliminate the uncertain effects of bias and confounding* which plague most other epidemiological studies. Any good textbook ${ }^{1}$ will give a formula for an RCT as follows: select a sufficient number of fairly representative patients, randomly allocate to two or more treatment groups, follow them all up (or as many as possible), over an appropriate time period, ensuring any assessment of outcome is masked to (i.e. independent of) knowledge of the treatment group. Although theoretically simple, in practice such studies are not always straightforward, often because of ethical considerations.

Depending on the study, concern usually centres on the ethics of denying/administering treatment to half the patients. Random allocation of treatment in an RCT requires explicit acknowledgement of the fact that it is not known which treatment is more effective; this may challenge deeply held beliefs derived from clinical experience or previous teaching. However, if there is no clinical consensus, with the result that a person may be treated differently depending on which physician they consult, an RCT replicates what is effectively random delivery of healthcare (at least from the patient's point of view) and also takes the practice of medicine one small step further. Systematic appraisal of scientific evidence and proper design, execution and reporting of trials is also a question of ethics; deficits in these research activities may adversely affect patient care.

The scientific approach to testing a hypothesis about treatment involves excluding chance, bias and confounding as possible explanations of any appar-

\footnotetext{
*Bias may be defined as any systematic error in a study that results in an incorrect result; confounding occurs when the groups being compared differ with respect to another factor or 'confounder' which is associated with the outcome (or disease) under study. Both bias and confounding can be thought of as alternative explanations for the observed effect.

Correspondence to: Jennifer Evans, Moorfields Eye Hospital, City Road, London EC1V 2PD,UK.
}

ent beneficial (or detrimental) effect. An RCT of adequate size enables measurement of relative effect, allowing for statistical evaluation of the role of chance in producing that effect; proper randomisation (of a sufficiently large number of people) will, by definition, eliminate confounding; and careful, independent 'masked' measurement of outcome reduces bias.

Nowadays, most clinicians and researchers should be well aware that trials which are too small are likely to be misleading. Depending on the treatment effect studied, a trial that is too small may suggest that there is no evidence that the two treatments are different, even if they really are different. On the other hand, if a statistically significant result is found in a small study, the estimate of treatment effect may be exaggerated. There is also a tendency for publication bias; 'significant' results are more likely to be submitted and accepted for publication, unless the trial is of sufficient size and stature for the result to be published whatever the result. ${ }^{2}$ This publication bias poses serious problems for people undertaking systematic reviews or meta-analyses of RCTs, which is why considerable effort is now being devoted to the development of complete registers of clinical trials. ${ }^{3}$ Calculations of the required sample size are relatively straightforward but need to be done at the design stage.

The aim behind randomisation (or random allocation of treatment) is to produce comparable treatment and control groups. If it is effective, both known and unknown confounders will be equally distributed between the two groups. This is the reason why the RCT is such a powerful study design - other epidemiological studies depend on the measurement and statistical analysis of known confounders. It is increasingly recognised that statistical techniques may not always control confounding adequately, especially if the confounding factor is not measured very well. ${ }^{4}$ Powerful biases can be introduced into non-randomised studies, or indeed into randomised studies, if clinicians are aware of the treatment group to which the next 
patient is to be allocated. ${ }^{5}$ An appreciation of the purposes behind, and the importance of, effective randomisation is critical, not only for evaluating the results of RCTs but also for understanding the difficulties attached to the interpretation of observational non-randomised studies.

Concealing the group to which each patient has been allocated is an effective way of avoiding bias in assessment of outcome. Placebos or use of similarlooking treatments aim to conceal the identity of different treatments both from the patient and from the person assessing outcome. Such masking is problematic in surgical trials and investigators need to be aware of the possible biases which may arise.

Whilst RCTs are not required to assess all forms of medical and surgical treatment, if there is real doubt as to the relative effectiveness of different treatments, an RCT, by helping to resolve uncertainty, will be the best way to minimise the number of patients exposed to a possibly inferior treatment. For example, there are currently several RCTs of the safety and effectiveness of intraocular lenses in developing countries. Although posterior chamber lenses are preferred by ophthalmologists in industrialised countries, the benefits of changing from intracapsular to extracapsular extraction are not clear cut in the context of high-volume, low-cost cataract surgery. ${ }^{6}$ In Nepal, an RCT is being used to assess the effectiveness of using anterior-chamber lenses, which are compatible with intracapsular cataract extraction, as an altenative to correction with aphakic spectacles. ${ }^{7}$ The study includes an assessment of the impact on quality of life from the patient's viewpoint, and an economic analysis to evaluate cost-effectiveness. This is an example of a surgical situation where, by providing best estimates of relative safety, effectiveness and acceptability, a timely RCT may help in the search for better ophthalmic health care.

\section{REFERENCES}

1. Pocock SJ. Clinical trials: a practical approach. Chichester: Wiley, 1983.

2. Simes RJ. Publication bias: the case for an international registry of clinical trials. J Clin Oncol 1986;4:1529-41.

3. Chalmers I, Dickersin K, Chalmers TC. Getting to grips with Archie Cochrane's agenda: all randomised controlled trials should be registered and reported. BMJ 1992;305:786-8.

4. Leon DA. Failed or misleading adjustment for confounding. Lancet 1993;342:479-81.

5. Chalmers TC, Celano P, Sacks HS, Smith H. Bias in treatment assignment in controlled clinical trials. $\mathrm{N}$ Engl J Med 1983;309:1358-61.

6. WHO. Use of intraocular lenses in cataract surgery in developing countries: memorandum from a WHO meeting. Bull WHO 1991;69:657-66.

7. Lahan IOL Study Group. Prospective randomised trial of anterior chamber lenses compared to conventional surgery in Nepal. Manual of operations. London: ICEH, 1991 (unpublished). 